\title{
TeleComVis: Exploring Temporal Communities in Telecom Networks
}

\author{
Qi Ye, Bin Wu, Lijun Suo, Tian Zhu, Chao Han, and Bai Wang
}

Beijing Key Laboratory of Intelligent Telecommunications Software and Multimedia Beijing University of Posts and Telecommunications, Beijing, China, 100876

yeqibupt@gmail.com, \{wubin, wangbai\}@bupt.edu.cn

\begin{abstract}
By the structure of call graphs derived from huge amounts of Call Detail Records, we can find out the social communities in the call graphs and make different market strategies for these social communities in real telecom applications. However, traditional telecom business intelligence methods are short of ways to understand the social interactions. To fill this gap, we propose a Telecom Community Visual Analysis prototype tool, called TeleComVis, to analyze the call graphs derived from Call Detail Records. In the demo, we will show (1) the functions of TeleComVis; (2) the critical techniques of finding statistically significant communities in real-world telecom applications. Using TeleCom Vis, users can both analyze the statistical properties of massive call graphs and explore the statistically significant communities and the temporal links interactively.
\end{abstract}

Keywords: Visual Analytics, Community, Call Graph, Social Network.

\section{Introduction}

Nowadays, researchers are increasingly interested in addressing a wide range of challenges residing in social networks. Taking a social network analysis and visual analytics approach, based on the framework of JSNVA [1, we develop a tool called TeleCom Vis in Java programming language to analyze the structure of massive call graphs derived from Call Detail Records (CDR) and the relationships among customers. Currently, many famous social network visualization tools have been proposed, such as Vizster [2]. To overcome the problem of scalability, Vizster allows users to explore massive social networks through egocentric networks. In real telecom applications, there are two scenarios that are not well addressed by current available visual analytical tools: one is how to detect the statistically significant communities accurately and interactively, and the other is how to show the temporal comparison of the call patterns.

\section{Tool-TeleCom Vis}

TeleCom Vis keeps three data structures for graphs: the raw graph which provides the structure of original graph, the subgraph which contains a subgraph in 
the raw graph and the community graph which is an abstract graph derived from the raw graph in which each vertex is a community and the edges indicate the relationships between communities. TeleCom Vis classifies the operations into the following steps: preprocessing, topological statistical analysis, community detection and visual analysis.

\subsection{Preprocessing and Statistical Analysis}

The 'Preprocessing' tab enables users to load network data into TeleCom Vis, as shown in Fig. 1 (a). Once the graph file has been imported, users can import the weight or temporal information of the edges. In the temporal information file, each edge has a natural number $s \in \mathbb{N}$ standing for a time step in which the edge is active. The 'Statistics' tab enables users to use different network algorithms to get the topological statistical properties of the raw graph. As shown in Fig. 1 (b), there are several typical network analysis methods to get the statistical properties of the whole graph, such as degree distribution, component distribution, cluster coefficient, maximal clique distribution, etc. To measure the relative importance of customers in call graphs, various centrality algorithms have also been provided. TeleCom Vis also provides several algorithms to sample the subgraph from the raw graph.

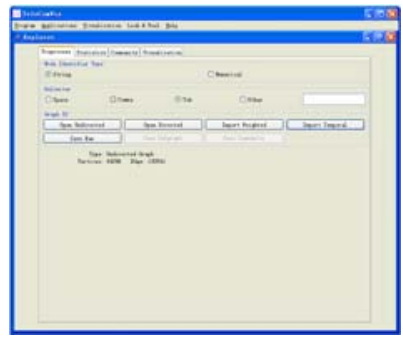

(a) Preprocessing

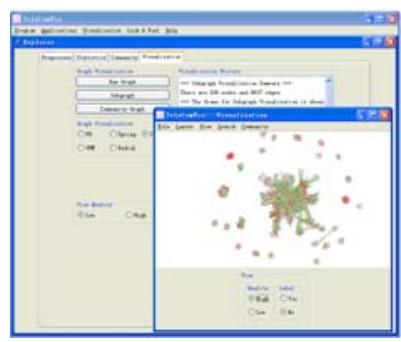

(d) Visual Analysis

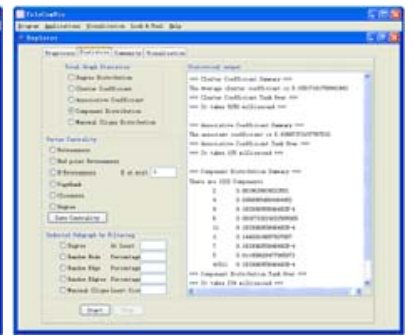

(b) Statistical Analysis

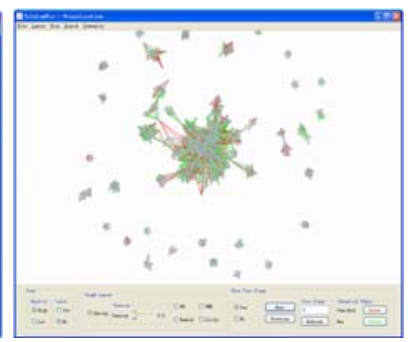

(e) Subgraph Visualization

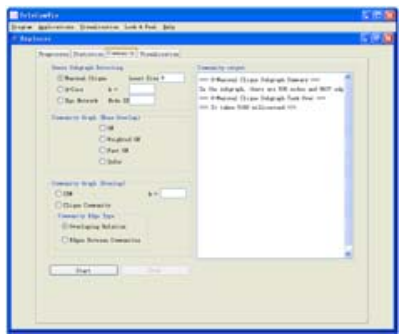

(c) Community Detection

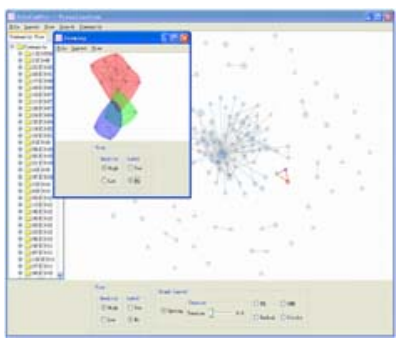

(f) Community Overview

Fig. 1. TeleCom Vis user interface 


\subsection{Community Detection}

The 'Community' tab enables users to get the dense subgraphs or communities from the original raw graph, as shown in Fig. 1(c). To get an overview of massive graphs, one practical strategy is to show the cohesive subgraphs. We call the maximal cliques which contain at least $k$ vertices as $k$-maximal cliques. We provide $k$-maximal clique enumeration algorithm and $k$-core algorithm to explore the dense subgraph in call graphs. TeleComVis can enumerate all the maximal cliques in a call graph with tens of thousands of vertices and edges in just several seconds which makes it possible to explore real-world call graphs interactively.

In TeleComVis, we provide two types of community graph to be analyzed: separated communities and overlapping communities, such as CPM [3]. There are two types of edges between overlapping communities: one shows the connection relationships and the other one shows the overlapping relationships. Fig[1(f) shows the 8-clique-community graph of a mobile call graph with tens of thousands of vertices and hundreds of thousands of edges using the CPM algorithm.

\subsection{Visual Analysis}

As shown in Fig. 1 (d), the 'Visualization' tab enables users to show the raw graph, subgraph and community graph in new network visualization frames, respectively. As shown in Fig. 1.(d), (e) and (f), in the network visualization

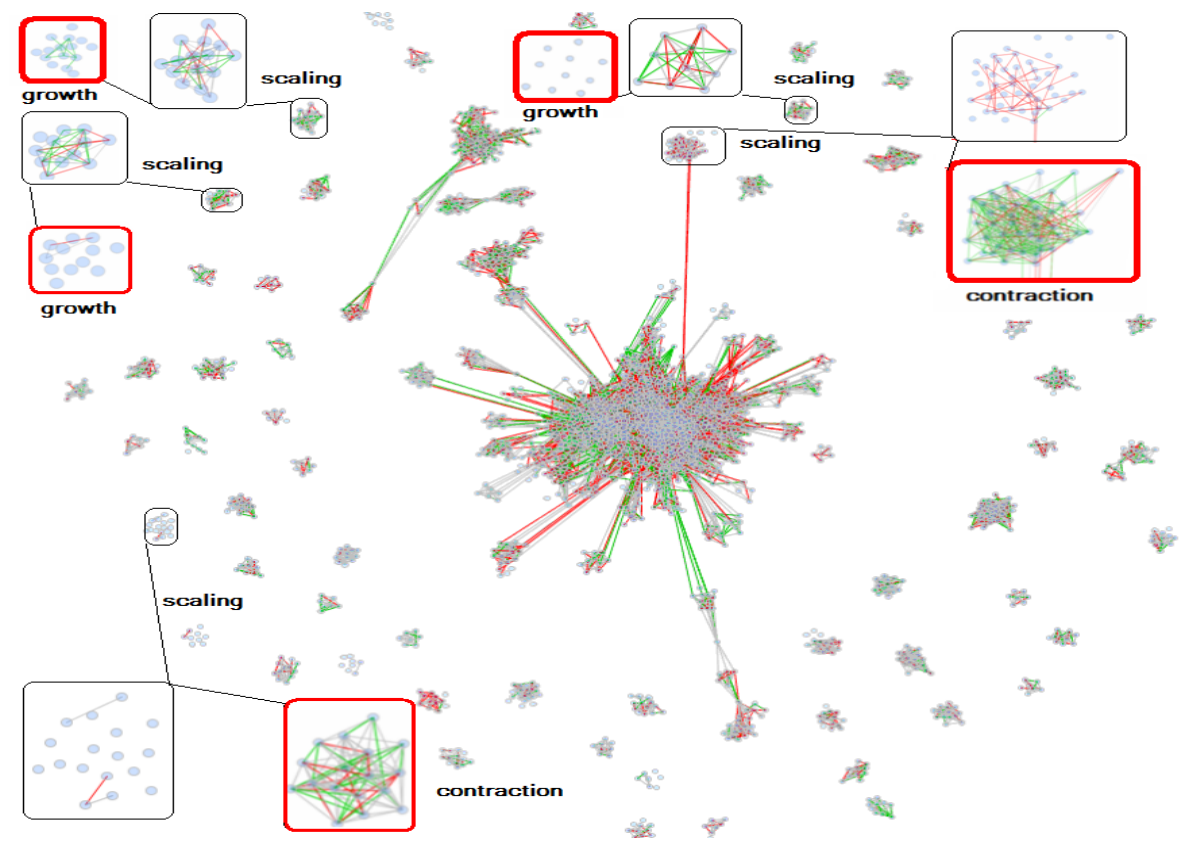

Fig. 2. The evolution of 8-maximal cliques in a mobile call graph in the last month 
frame, basic interaction is done with simple mouse operations. Dragging the mouse to select a group of community vertices causes these communities to automatically expand, and users can zoom into the overlapping relationships between actors. They can also drag the vertex in the panel to change its position. It also provides better capabilities to manipulate graph display details, such as labeling, color coding, size normalization and scaling. As show in Fig. 1] (e) and (f), different graph layout algorithms in the framework JSNVA 1 are offered, such as FR layout algorithm, n-body based spring algorithm [2], radial algorithm, etc.

To show temporal links, we define 3 types of edges: the persistent edges which appear both in previous and current time steps, the vanished edges which appear in previous time step but disappear in current time step and the new born edges which do not appear in previous time step but appear in current time step. Fig. 2 shows the dynamical patterns of the subgraph made by 8-maximal cliques in a temporal mobile call graph in a city of China during 7 months which has 49035 vertices and 158543 edges. The grey edges indicate the persistent edges, and the red edges indicate the vanished edges, and the green edges indicate the new born ones. Fig. 2 shows the structure of the subgraph in the last month. To show temporal links, the link relationships in the second month are shown in the red frame.

\section{Summary}

By combining the techniques of social network analysis and visual analytics, we develop TeleComVis to characterize the relationships between customers and their social groups in massive call graphs. By allowing different social network analysis methods and fast community detection algorithms, we believe that these techniques can reduce unnecessary interaction and make visual analysis of temporal telecom call data more effectively.

Acknowledgments. Supported by the National Natural Science Foundation of China under Grant No. 60402011, the National Key Technology R\&D Program of China under Grant No.2006BAH03B05, and the Specialized Research Fund for the Joint laboratory between Beijing University of Posts and Communications and IBM China Research Laboratory (No.JTP20071002-4, JTP200806014-3).

\section{References}

1. Ye, Q., Zhu, T., Hu, D., et al.: Cell Phone Mini Challenge Award: Exploring Temporal Communication in Mobile Call Graphs. In: 3rd IEEE Symposium on Visual Analytics Science and Technology, pp. 207-208. IEEE Press, Columbus (2008)

2. Heer, J., Boyd, D.: Vizster: Visualizing online social networks. In: 9th IEEE Symposium on Information Visualization, pp. 32-39. IEEE Press, Minneapolis (2005)

3. Palla, G., Derényi, I., Farkas, I., Vicsek, T.: Uncovering the overlapping community structure of complex networks in nature and society. Nature 435, 814-817 (2005) 